\title{
Long-lasting desynchronization in rat hippocampal slice induced by coordinated reset stimulation
}

\author{
P. A. Tass, ${ }^{1,2}$ A. N. Silchenko, ${ }^{1}$ C. Hauptmann, ${ }^{1}$ U. B. Barnikol, ${ }^{1,2}$ and E.-J. Speckmann ${ }^{3}$ \\ ${ }^{1}$ Institute of Neuroscience and Medicine-Neuromodulation (INM-7) and Virtual Institute of Neuromodulation, \\ Research Center Jülich, D-52425 Jülich, Germany \\ ${ }^{2}$ Department of Stereotaxic and Functional Neurosurgery, University of Cologne, D-50931 Cologne, Germany \\ ${ }^{3}$ Institute of Physiology, University of Münster, D-48149 Münster, Germany \\ (Received 25 September 2008; revised manuscript received 8 April 2009; published 2 July 2009)
}

\begin{abstract}
In computational models it has been shown that appropriate stimulation protocols may reshape the connectivity pattern of neural or oscillator networks with synaptic plasticity in a way that the network learns or unlearns strong synchronization. The underlying mechanism is that a network is shifted from one attractor to another, so that long-lasting stimulation effects are caused which persist after the cessation of stimulation. Here we study long-lasting effects of multisite electrical stimulation in a rat hippocampal slice rendered epileptic by magnesium withdrawal. We show that desynchronizing coordinated reset stimulation causes a long-lasting desynchronization between hippocampal neuronal populations together with a widespread decrease in the amplitude of the epileptiform activity. In contrast, periodic stimulation induces a long-lasting increase in both synchronization and amplitude.
\end{abstract}

DOI: 10.1103/PhysRevE.80.011902

PACS number(s): 87.19.L-, 05.40.Ca, 05.45.Xt, 84.35.+i

Synchronization is a classical self-organization phenomenon [1]. Abnormally strong neuronal synchronization is, e.g., found in epilepsy [2]. Experiments have been devoted to suppress epileptiform activity by continuous or adaptive pulsatile or smooth electrical stimulation [3]. Reinitiation of epileptiform activity follows stimulus removal immediately or after a few minutes (e.g., 4 min) [3]. In contrast, our modelbased approach does not aim at a suppression of epileptiform activity during stimulation, but at a long-lasting change in the network's dynamics due to a stimulation-induced reshaping of the network connectivity.

Network topology strongly impacts on network dynamics [4]. Unlike in many physical systems, in biological neural networks the (coupling) strength of a synapse (conveying signals from a presynaptic to a postsynaptic neuron), is not constant, but depends on the neuronal timing [5]: if a presynaptic spike advances the postsynaptic spike, the synaptic weight increases, whereas in the opposite case the synaptic weight decreases. This mechanism, the spike timingdependent plasticity (STDP), is fundamental to learning and memory in nervous systems [5]. Dynamical and connectivity changes are tightly connected in neuronal populations [6]. Neural and phase oscillator networks with STDP are multistable [7]: the attractors differ in their fast neuronal dynamics and their slow synaptic dynamics (connectivity pattern). Stable states with stronger synchronization and stronger average synaptic weight coexist with stable desynchronized states with a weaker average synaptic weight [7].

As shown numerically, the tight relationship between network dynamics and connectivity enables to reshape the connectivity by controlling the dynamics. Stimulation techniques may shift a network from one attractor to another, so that the stimulation effect outlasts stimulus offset: the network learns or unlearns synchrony $[7,8]$. Periodic stimulation increases the amount of synchrony along with the rate of coincidences and the mean synaptic weight. The network is shifted from a desynchronized state with weak coupling to a synchronized state with strong coupling [7]. Conversely, desynchronizing stimulation, e.g., coordinated reset (CR) stimulation [9], decreases the rate of coincidences and the synaptic weights. The network is shifted from a synchronized state with strong coupling to a desynchronized state with weak coupling [7].

We present an experimental study of long-lasting CR stimulation effects. We applied multisite electrical stimulation to the low-magnesium $\left(\left[\mathrm{Mg}^{2+}\right]\right.$-free $)$ model of epileptiform activity in a rat hippocampal slice [10]. This welldefined experimental model of seizurelike activity exhibits robust neuronal synchronization compared to, e.g., the $\left[\mathrm{Ca}^{2+}\right]$-free or bicuculine models [11]. We show that CR stimulation induces a long-lasting desynchronization between hippocampal neuronal populations and a widespread decrease in the amplitude of the epileptiform activity. In contrast, periodic stimulation induces a long-lasting increase in synchronization as well as amplitude.

Slices from rat hippocampus were kept in $\left[\mathrm{Mg}^{2+}\right]$-free artificial cerebrospinal fluid (ACSF) to evoke epileptiform field potentials (sharp waves followed by slow oscillations) with a mean frequency of about $0.15 \mathrm{~Hz}$ [12] [Fig. 1(b)], which occurred spontaneously (without stimulation) and persisted over hours without changes in amplitude and frequency [10]. Recording sites: we recorded local-field potentials (LFPs) in the CA1 area with a linear array of four insulated stainless steel electrodes (with an interelectrode distance of $100 \mu \mathrm{m}$ ), positioned perpendicularly to the stratum (str.) pyramidale along the apical dendrites of the principal cells, reaching from the stratum oriens (LFP 1), via the thin stratum pyramidale to the broad stratum radiatum (LFPs 2-4) [Fig. 1(a)]. LFP recordings were monopolar, with a distant common reference in the ACSF. Stimulation sites: stimulation in $\mathrm{CA} 3$ activates predominately the stratum radiatum of CA1 [14]. A linear array of four stainless steel macroelectrodes (with an interelectrode distance of $200 \mu \mathrm{m}$ ) was placed in the Schaffer collaterals which excitatorily project from CA3 to all types of neurons in CA1 [14] [Fig. 1(a)].

To study the effects that outlast the cessation of stimulation, we compared the dynamics before and after stimulation. First, during $30 \mathrm{~min}$ we recorded the spontaneous LFPs in 


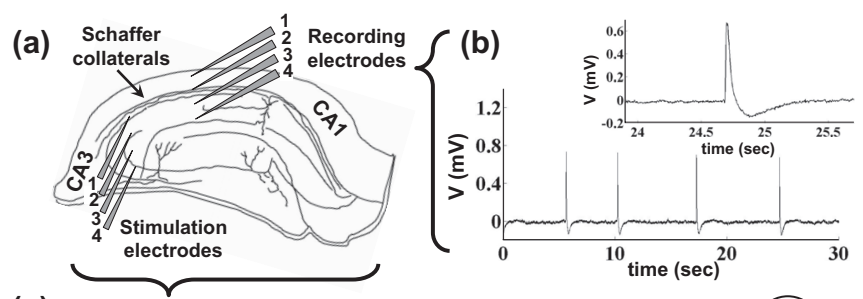

(c)

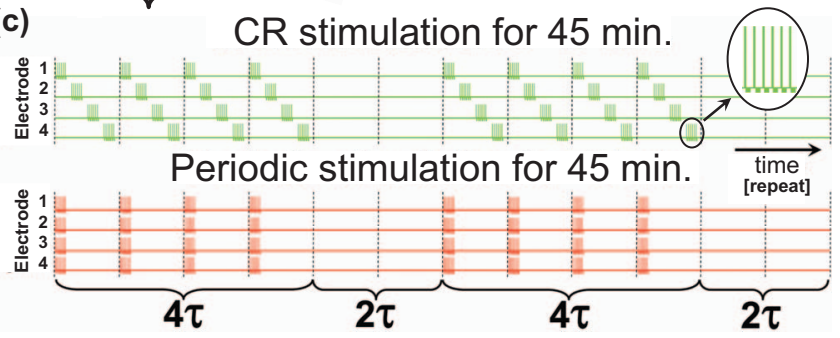

FIG. 1. (Color) (a) Stimulation electrodes were placed in CA3 of rat hippocampal slice, projecting to CA1 via the Schaffer collaterals. Recording electrodes were placed in CA1. (b) Typical time course of epileptiform discharges of CA1. (c) CR and periodic stimulations: diagrams of the four-site stimulation pattern intended to sequentially $(\mathrm{CR})$ or simultaneously (periodic stimulation) reset the phases of four neuronal populations in CA1. For both stimulation types in each slice the stimulation period $\tau$ was adapted to the prestimulus mean period $T$ of the epileptiform discharges. 4 cycles with stimulation $(\mathrm{ON})$ were followed by 2 cycles without stimulation (OFF) [13]. The 4 cycles $\mathrm{ON}-2$ cycles $\mathrm{OFF}$ sequence was periodically repeated at intervals of $6 \tau$.

CA1. Then, multisite electrical stimulation was performed during 45 min. Finally, we measured the LFPs in CA1 during 50 min after stimulation. We used two multisite stimulation protocols [Fig. 1(c)]: (1) CR, where a sequence of resetting pulse trains is administered one after another via electrodes 1-4 with a time delay of a fourth of the mean duration of the prestimulus seizurelike oscillation [9]. Each pulse train consisted of six charge balanced pulses with an intraburst frequency of $120 \mathrm{~Hz}$, an amplitude of $1-1.75 \mathrm{~V}$, and a duration of $0.15 \mathrm{~ms}$. (2) As a control we used periodic stimulation with bursts delivered via all four sites simultaneously. For $\mathrm{CR}$ and periodic stimulations, we adapted our stimulation period $\tau$ to the prestimulus mean period $T$ of the epileptiform activity of the particular slice (ranging from 4 to $10 \mathrm{~s}$ ) and used a periodic sequence: four stimulation cycles were followed by 2 cycles pause [Fig. 1(c)]. Hence, the current delivery was normalized to $T$, and the only difference between $\mathrm{CR}$ and periodic stimulations was the sequential vs the simultaneous reset. We tested CR and periodic stimulations in three different hippocampal slices each.

The epileptiform activity was suppressed by CR stimulation, slowly re-emerged after CR offset and remained reduced in the entire poststimulus epoch [Fig. 2(a)]. In contrast, the epileptiform activity was boosted by periodic stimulation and remained enhanced in the entire poststimulus epoch [Fig. 2(b)]. To quantify the stimulation effects on the amplitudes and phases of different frequency bands, we used a wavelet-based phase synchronization and an amplitude analysis (cf. [15]). The analytical wavelet transformation of an LFP signal $x(t)$ reads $W(s, t)=\frac{1}{\sqrt{s}} \int_{-\infty}^{+\infty} x(\xi) \psi^{*}[(\xi-t) / s] d \xi$, where $\psi_{s, t}$ is the mother-wavelet function, which is the Mor-
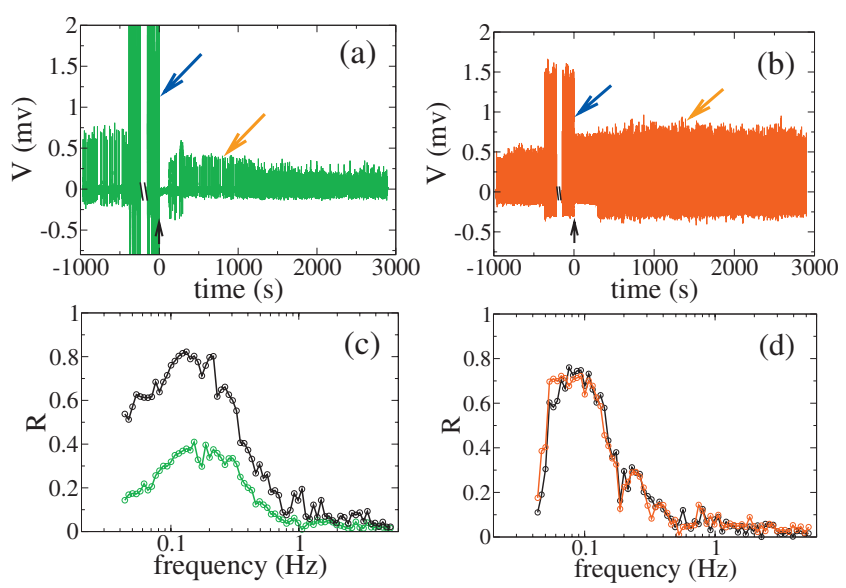

FIG. 2. (Color) LFP 1 (from electrode 1) from CA1 before, during (partly compressed) and in the entire interval after (a) CR and (b) periodic stimulation. Black arrows indicate stimulation offsets. Synchronization index between LFP 1 and LFP 2 from CA1 before (black line) and after (green/red line) (c) CR and (d) periodic stimulation. Results for pairs LFP 1/LFP 3 and LFP 1/LFP 4 were similar. Stimulation artifacts (blue arrows) were caused by direct pickup of stimulation-induced potentials in the extracellular space and exceeded the epileptiform activity (orange arrows).

let wavelet with central frequency $\sigma=5.336446$ [16]. At any time $t$ and for every time scale $s$, the analytical wavelet transform $W(s, t)=E(s, t) \exp \left[J \phi_{s}(t)\right]$ provides the amplitude $E(s, t)=\sqrt{\operatorname{Re}[W(s, t)]^{2}+\operatorname{Im}[W(s, t)]^{2}}$ and the phase $\phi_{s}(t)$ $=\arctan \{\operatorname{Im}[W(s, t)] / \operatorname{Re}[W(s, t)]\}$ of signal $x(t)[16]$. The relation between the time scale $s$ of the wavelet transform and the corresponding frequency $f$ of the Fourier transform reads $s \cong 1 / f$.

To study the phase synchronization of two LFP signals $x_{1}(t)$ and $x_{2}(t)$, for every time scale $s$, we determined the
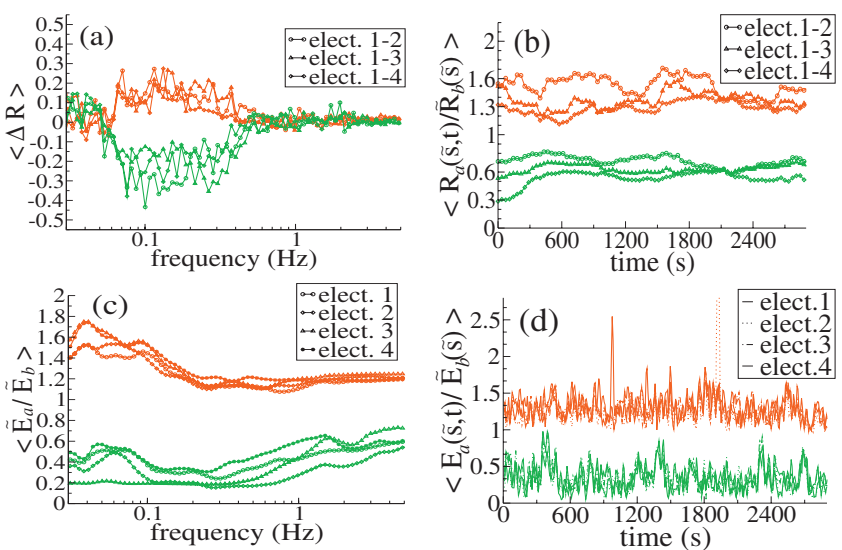

FIG. 3. (Color) (a) Differences of synchronization before and after stimulation $\langle\Delta R(f)\rangle$; (b) time-dependent poststimulus indexes $R_{a}(\hat{s}, t)$ in the frequency band around $\hat{f}=0.12 \mathrm{~Hz}$, normalized to their prestimulus means $\bar{R}_{b}(\hat{s})$; (c) poststimulus amplitudes $\bar{E}_{a}$ normalized to their prestimulus means $\bar{E}_{b}$; (d) time-dependent poststimulus amplitudes $\bar{E}_{a}(\tilde{s}, t)$ around $\tilde{f}=0.22 \mathrm{~Hz}$, normalized to their prestimulus means $\bar{E}_{b}(\widetilde{s})$. Brackets denote averaging over three slices. Green curves refer to $\mathrm{CR}$; red curves refer to periodic stimulation. 
instantaneous phases $\phi_{s, 1}, \phi_{s, 2}$ of $x_{1}(t), x_{2}(t)$ and the phase difference $\Delta \phi_{s, 1,2}=\phi_{s, 1}-\phi_{s, 2}$. Phase synchronization between the LFPs showed up as the presence of one prominent peak in the distribution of $\Delta \phi_{s, 1,2}$ and was quantified during the spontaneous $30 \mathrm{~min}$ epoch before and the $50 \mathrm{~min}$ epoch after stimulation with the synchronization index $R(s)$ $=\sqrt{\left\langle\cos \left(\Delta \phi_{s, j, k}\right)\right\rangle^{2}+\left\langle\sin \left(\Delta \phi_{s, j, k}\right)\right\rangle^{2}}[17]$ for each time scale $s$ for all pairs of electrodes $j, k=1, \ldots, 4(j \neq k) .\langle\cdots\rangle$ denotes averaging over the prestimulus or the poststimulus epoch.

In all slices we revealed a strong mutual phase synchronization of the epileptiform activity of LFPs 2, 3, and 4 in CA1 both before and after stimulation. In particular, this type of synchronization was neither significantly affected by CR nor by periodic stimulation. In contrast, the phase synchronization of the epileptiform activity between LFP 1 on one hand and LFPs 2-4 on the other hand was strongly affected: CR stimulation caused a pronounced decrease in the phase synchronization between LFP 1 and the other LFPs [Fig. 2(c)]. In contrast, the synchronization between LFP 1 and the other LFPs was not affected [Fig. 2(d)] or even increased [see Fig. 3(a)] due to periodic control stimulation. Results from two slices are shown in Fig. 2.

To show consistency across slices, we used the mean difference $\langle\Delta R(f)\rangle=\left\langle R_{a}(f)-R_{b}(f)\right\rangle$ of the frequency-dependent synchronization indices $R(f)$ and the mean ratio of the average amplitudes $\left\langle\hat{E}_{a}(f) / \hat{E}_{b}(f)\right\rangle$ in different frequency bands calculated ("a") in the 50 min window after and ("b") in the 30 min window before stimulation, where $\langle\cdot\rangle$ stands for averaging over three slices. Intriguingly, the effects of the two stimulation types on LFP amplitudes and on the interaction between superficial (stratum oriens) and deeper (distal stratum radiatum) epileptiform activities were diametrical and reproducible:

(1) Synchronization between LFPs: in all slices, CR stimulation decoupled the superficial LFP 1 from all other deeper LFPs 2-4 [Fig. 3(a)], mainly in the burst discharge range $(0.1-0.25 \mathrm{~Hz})$. In contrast, the periodic stimulation increased the synchronization between LFP 1 and the deeper LFPs 2-4 in that frequency range [Fig. 3(a)]. CR and periodic stimulation did not significantly change the synchronization between the deeper LFPs 2-4.

(2) Amplitude of LFPs: CR stimulation decreased the amplitudes of all LFPs in a wide frequency range from $0.1 \mathrm{~Hz}$ to more than $1 \mathrm{~Hz}$ [Fig. 3(b)], whereas periodic stimulation increased all LFP amplitudes [Fig. 3(b)].

In all slices the effects on synchronization and amplitude were most pronounced at around $\hat{f}=0.12 \mathrm{~Hz}$ and $\tilde{f}$ $=0.22 \mathrm{~Hz}$, respectively. We calculated the corresponding time-resolved synchronization index $R_{a}(\hat{s}, t)$ in a sliding 225 $\mathrm{s}$ window and normalized it by the mean value in the $30 \mathrm{~min}$ prestimulus window $\bar{R}_{b}(\hat{s}) .\left\langle R_{a}(\hat{s}, t) / \bar{R}_{b}(\hat{s})\right\rangle$ is the average across three slices. Analogously, we calculated $\left\langle E_{a}(\tilde{s}, t) / \bar{E}_{b}(\widetilde{s})\right\rangle$, the time-dependent normalized wavelet amplitudes averaged across three slices. The diametrical effects of CR and periodic stimulations on LFP synchronization [Fig. 3(c)] and LFP amplitudes [Fig. 3(d)] persisted in the entire $50 \mathrm{~min}$ poststimulus epoch.

We revealed two effects of CR stimulation: (i) a widespread amplitude decrease in LFPs 1-4. As LFPs reflect neural mass activity [18], this amplitude decrease may likely correspond to an overall decrease in synchrony in several layers of CA1, ranging from str. (stratum) oriens to pyramidale. In future studies this process may be analyzed at higher spatial resolution by cellular measurements (micropipette recordings) or optical imaging. (ii) A pronounced decoupling between str. oriens (LFP 1) and str. pyramidale through distal str. radiatum (LFPs 2-4), but no relevant decoupling between LFPs 2-4. This differential decoupling may have two reasons: (i) the projection fields of the Schaffer collaterals belonging to the different stimulation electrodes (Fig. 1) have a small overlap in str. oriens, but a strong overlap in the deeper layers (LFPs 2-4) [14]. Accordingly, stimulation of str. oriens was spatially selective, while the deeper layers (LFPs 2-4) were affected more diffusively and, hence, reset as a whole. (ii) The connectivity in str. oriens and in the deeper layers is different. Even different populations of inhibitory interneurons are found in str. oriens (interneurons at the str. oriens/alveus border) and str. pyramidale (chandelier and basket cells) [14]. Accordingly, due to their connectivity the superficial (LFP 1) and the deeper layers (LFPs 2-4) might act as separate neuronal clusters. One approach to dissect these two factors might be to stimulate CA1 directly.

In noisy (computational) networks with fixed strong coupling desynchronized states are typically unstable [1,7]. Yet, to make the network unlearn synchrony, we control the slow synaptic dynamics and eventually reach a favorable basin of attraction with desynchronization and weak connectivity [7]. Our experimental results on long-lasting (de)synchronization fit to theoretical predictions on CR and periodic stimulations [7]. Possible connectivity changes, e.g., a decline of synaptic receptor density [19] and an emergence of new synaptic connections $[6,20]$, have to be analyzed with different experimental techniques. We anticipate connectivity changes because network connectivity and dynamics are tightly connected [6], and because short pilot stimulation trials of up to $10 \mathrm{~min}$ duration were ineffective, indicating a slow synaptic relearning. Our approach might offer a technology for seizure control inducing a long-lasting desynchronization.

We thank B. Herrenpoth, L. Sasse (Institute of Physiology, University of Münster), and the Network of Excellence in Biosimulation (Grant No. LSHB-CT-20004-005137). 
[1] H. Haken, Advanced Synergetics (Springer, Berlin, 1983); A. S. Pikovsky et al., Synchronization: A Universal Concept in Nonlinear Science (Cambridge University Press, Cambridge, UK, 2001).

[2] Epilepsy as a Dynamic Disease, edited by J. Milton and P. Jung (Springer, Berlin, 2003).

[3] S. M. Bawin et al., Brain Res. 399, 194 (1986); K. Jerger and S. J. Schiff, J. Neurophysiol. 73, 876 (1995); B. J. Gluckman et al., ibid. 76, 4202 (1996); R. J. Warren and D. Durand, Brain Res. 806, 186 (1998); M. Bikson et al., J. Physiol. (London) 531, 181 (2001); B. J. Gluckman et al., J. Neurosci. 21, 590 (2001); J. Lian et al., J. Physiol. (London) 547, 427 (2003).

[4] J. G. Restrepo, E. Ott, and B. R. Hunt, Phys. Rev. Lett. 96, 254103 (2006); I. Belykh, E. de Lange, and M. Hasler, ibid. 94, 188101 (2005); S. H. Strogatz, Nature (London) 410, 268 (2001).

[5] W. Gerstner et al., Nature (London) 383, 76 (1996); H. Markram et al., Science 275, 213 (1997); G. Q. Bi and M. Poo, J. Neurosci. 18, 10464 (1998); G. Wittenberg and S. Wang, ibid. 26, 6610 (2006).

[6] F. Engert and T. Bonhoeffer, Nature (London) 399, 66 (1999); R. Yuste and T. Bonhoeffer, Nat. Rev. Neurosci. 5, 24 (2004).

[7] P. A. Tass and M. Majtanik, Biol. Cybern. 94, 58 (2006); P. A. Tass and C. Hauptmann, Int. J. Psychophysiol. 64, 53 (2007); C. Hauptmann and P. A. Tass, BioSystems 89, 173 (2007); Y. L. Maistrenko, B. Lysyansky, C. Hauptmann, O. Burylko, and P. A. Tass, Phys. Rev. E 75, 066207 (2007).

[8] Multistability also emerges in oscillator networks with a symmetrical Hebbian synaptic learning rule [P. Seliger, S. C. Young, and L. S. Tsimring, Phys. Rev. E 65, 041906 (2002)]. The stimulation induced switching between synchronized and desynchronized states works here, too [7].

[9] P. A. Tass, Biol. Cybern. 89, 81 (2003).

[10] W. W. Anderson et al., Brain Res. 398, 215 (1986).

[11] H. L. Haas and J. G. R. Jefferys, J. Physiol. (London) 354, 185
(1984); H. E. Scharfman, Neuroscience 59, 245 (1994).

[12] Transverse rat hippocampal slices $(450-500 \mu \mathrm{m})$ were cut in parallel to the alvear fibers with a McIlwain tissue vibratom and preincubated in a submersion chamber for at least $1 \mathrm{~h}$ in $28{ }^{\circ} \mathrm{C} \mathrm{ACSF}$ with (mmol/l): $\mathrm{NaCl} 124 ; \mathrm{KCl} 4 ; \mathrm{CaCl}_{2} 1$; $\mathrm{NaH}_{2} \mathrm{PO}_{4}$ 1.24; $\mathrm{MgSO}_{4}$ 1.3; $\mathrm{NaHCO}_{3}$ 26; glucose 10. The ACSF was equilibrated with $5 \% \mathrm{CO}_{2}$ in $\mathrm{O}_{2}(\mathrm{pH}=7.4)$. Slices were transferred to a recording submersion chamber which was continuously perfused by ACSF ( $4 \mathrm{ml} / \mathrm{min}$; total bath exchange within $2 \mathrm{~min}$ ), and $\mathrm{pH}$ and temperature were continuously measured and stabilized at 7.4 and $32{ }^{\circ} \mathrm{C}$. The ionic composition of the ACSF was the same as during preincubation except for the $\mathrm{Ca}^{2+}$ concentration which was elevated to $2.0 \mathrm{mmol} / 1$.

[13] As shown numerically, during the ON cycles a four-clusterstate emerges, whereas during the OFF cycles the population transiently relaxes into a desynchronized state [9]. The OFF cycles improve the desynchronizing and the antikindling effects of CR stimulation [7]. In contrast, the kindling impact of periodic stimulation decreases with increasing OFF fraction [7].

[14] N. Ishizuka et al., J. Comp. Neurol. 295, 580 (1990); C. Bernard and H. V. Wheal, Hippocampus 4, 497 (1994).

[15] A. E. Hramov, A. A. Koronovskii, V. I. Ponomarenko, and M. D. Prokhorov, Phys. Rev. E 73, 026208 (2006).

[16] S. Mallat, A Wavelet Tour of Signal Processing (Academic, New York, 1997).

[17] P. Tass, M. G. Rosenblum, J. Weule, J. Kurths, A. Pikovsky, J. Volkmann, A. Schnitzler, and H. J. Freund, Phys. Rev. Lett. 81, 3291 (1998); M. Rosenblum et al., in Handbook of Biological Physics, edited by F. Moss (Elsevier, Amsterdam, 2000), p. 279.

[18] A. D. Legatt et al., J. Neurosci. Methods 2, 203 (1980).

[19] C. M. McCann et al., Nat. Neurosci. 11, 807 (2008).

[20] J.-V. Le Bé and H. Markram, Proc. Natl. Acad. Sci. U.S.A. 103, 13214 (2006). 